\title{
A Proposal for Reliable Information Multicast Environment : its Implementation and Evaluation
}

\author{
Osamu Takahashi*, Teruji Shiroshita*, Tetuo Sano*, \\ Masahide Yamashita*, Yukihiro Nakamura* and Masato \\ Maruyama**
}

*High-Speed Computer Networking Lab.

NTT Information and communication System Labs., NTT

1-2356 Take, Yokosuka, Kanagawa 238-03 Japan

TEL: +81-468-59-2415 FAX: +81-468-59-3796

E-mail:rmtp@isl.ntt.jp

**Multi-media System Group, NTT Electronics Technology

Corporation

3-12 Sakae-tyou, Kanagawa-ku, Yokohama, Kanagawa 221 Japan

\begin{abstract}
This paper proposes RIME (a Reliable Information Multicast Environment) which supports reliable multicast information delivery, error-free and secure, between a single server and thousands of remote clients. Using RIME it is possible to simultaneously 'multicast' such multimedia contents as data, text, image, video and even software updates over networks such as LAN, TCP/IP networks, ATM, ISDN, PSTN or the Internet.
\end{abstract}

Keywords

Reliable multicast, RMTP, RIME, reliable multicast transport protocol, MFDF

\section{INTRODUCTION}

The rapidly developing information age requires large-scale reliable information distribution systems. For example;

1) Newspaper multicast delivery by using a single server connected to a public network. It is possible to deliver to a large number of pre-registered clients, and charge 
after delivery.

2) Multicast software upgrades to numerous remote sites over the Internet while using only minimal human resources. This can lead to huge savings in the cost of dispatching engineers to make software upgrades in an installed base.

3) Multicasting game software or music videos error free.

All of these applications require that identical information be delivered to plural clients. 'Multicast (1 to n communication)' is essential to efficient distribution, because multicasting provides the function that can transmit information to plural clients simultaneously.

'Reliability' is also important because program files, data bases, etc. should be transmitted error free to the proper clients (subscribers). 'Reliable Multicast delivery' is necessary in order to deliver the information within the time needed and with adequate security, even in the case of large scale systems that have thousands of clients.

Almost all traditional computer applications are peer - peer (1 to 1) type communication, and reliability is realized by the OSI connection-mode protocols or the TCP/IP protocol suite. Some applications, such as MBone (Casner, 1992) for audio/video applications, use multicast technologies (protocols) but are not reliable. No reports or products have introduced multicast protocols that also reliable.

This paper proposes RIME, a Reliable Information Multicast Environment, which supports reliable multicast information delivery from a single server to thousands of remote clients. First, we discuss the requirements of the reliable multicast information delivery service. Second, we propose RIME and four types of tools. Third, we discuss the basic functions of RIME, such as the protocol stack and its software configuration. We propose the new multicast function MFDF (Multicast File Delivery Function) and the new protocol RMTP (Reliable Multicast Transport Protocol) for RIME. Fourth, we discuss the implementation of RIME and its performance. Experiments confirm that RIME can provide reliable multicast delivery with sufficient performance and stability. Finally, we discuss open issues and our future plans.

\section{REQUIREMENTS FOR RELIABLE INFORMATION MULTICAST ENVIRONMIENT}

New service that will deliver large amounts of information on-line have become feasible with the advent of high-speed networks which offer large bandwidth. On-line publishing services such as newspapers or magazines appear promising. In order to realize these services, a multicast communication environment must be developed that can delivery massive amounts of information to thousand of clients while satisfying the following requirements.

\section{a)Reliability}

$100 \%$ error free digital information delivery is mandatory, that is digital information such as structure encoded documents or computer programs need to be transferred to clients without a flaw. The delivery system must tolerate a network quality decline and exceptional conditions of clients such as temporary unavailability. Information should be delivered only to registered clients and charged after delivery, in the case of charged services. Security is a critical requirement especially when Satellite is used.

\section{b)Scalability}

The delivery server must support thousands of clients, and should deliver the information to the clients simultaneously. If the value of information decrease with time, fairness of delivery time is important. Amount of information should be several tens of megabytes. The distributed server configuration should be available, so that the delivery system can efficiently 
support any number of clients.

\section{c)Network applicability}

In order to realize an efficient delivery service system, the network should be selectable depending on amount of delivery information, and the topology of the clients. The delivery service should be usable with various existing and promising networks such as Leased line, Analog telephone line, ISDN, LAN, Satellite, CATV, ATM, etc.

\section{d)Performance}

Delivery must be completed within an appropriate time limit depending on service requirements. Delivery load should be kept within the limits that ensure system reliability.

\section{ARCHITECTURE FOR RIME}

The architecture of RIME was developed with the following concept. Use existing protocols and products as far as possible and the well known TCP/IP protocol stack. Support the tools suitable for various network and service requirements.

There are four tools of RIME and they are categorized into two groups: Type A and Type $\mathrm{B}$ depending on the function of the network. Type A tools are applicable to unicast networks such as Leased line, Analog telephone line, ISDN, Internet without multicast router, etc. Type $B$ tools are applicable to multicast networks such as Ethernet LAN, Internet with multicast router, Satellite, CATV, ATM point - multipoint connection, etc. The architecture of RIME including these tools is shown in Figure 1.

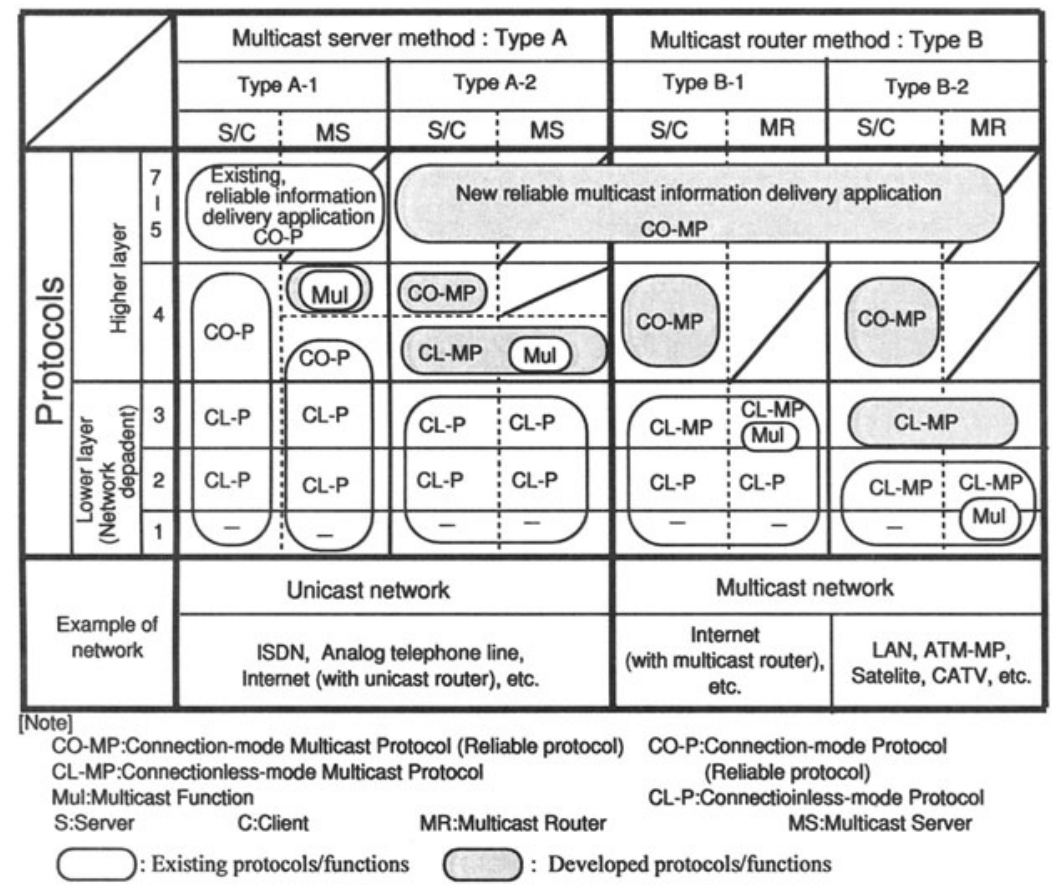

Figure 1 Architecture of RIME 


\subsection{Type A : Multicast Server method}

As Type A is applicable to unicast networks, the multicast function has to be realized in the multicast server site (Transport layer). So, we call Type A the Multicast Server method.

Type A includes two tools : Type A-1 and Type A-2. Type A-1 can use existing peer peer connection-mode application protocols and transport protocol without change. The multicast function is added to the Transport layer in the multicast server site. Reliability is realized by these existing connection-mode protocols, for example TCP (Postel, 1981). Type A-2 requires the multicast function in the Transport layer, new connection-mode multicast transport protocol and application protocols in both the server and clients site.

\subsection{Type B : Multicast Router method}

Type B is applicable to multicast networks but offer no reliability; this is achieved by RMTP (Shiroshita, 1996). Type B uses IP multicast protocol (Deering, 1989) as the network protocol, and requires multicast routers, so we call it the Multicast Router method. Because information delivery from the server basically finishes in one session, delivery time and server load are reduced dramatically. Type B includes two tools : Type B-1 and Type B-2. Type B1 realizes the multicast function at the network layer (router); Type B-2 realizes it under the data link layer such as shared media LAN, ATM Point - Multipoint connection and Satellite.

\section{SOFTWARE CONFIGURATION OF RIME}

Type A-1 and Type B-1 were implemented and evaluated. Software configurations are discussed in this section.

\subsection{Software configuration of Type A-1}

\subsubsection{Protocol Stack for Type A-1}

The FTP protocol stack was selected as the base protocol for Type A-1. FTP is a well known peer - peer protocol for file transfer (Postel 1985). A file can be transmitted to plural clients by repeated FTP transfer. However, it is not suitable for delivering a file to thousand of clients, because each FTP process imposes task switch overhead and file I/O overhead. In order to minimize the overheads, we added Multicast File Delivery Function (MFDF) on the TCP layer in the multicast server. The protocol stack for Type A-1 is shown in Figure 2.

\subsubsection{Software for Type A-1}

\section{(1)Basic function of MFDF}

1)FTP protocol processing

An FTP connection is established between the FTP server and FTP client, and uses two TCP connections, one for control, another for data transfer. MFDF realizes both FTP server and FTP clients functions. This means that the cascade interconnection of multicast servers is available if required.

\section{2)TCP connection management}

Many TCP connections are established concurrently between the multicast server and clients in the data transfer phase. The throughput of each TCP connection can vary because it 
depends on the performance of the clients' computers. If not all TCP connections can be managed individually, the completion of file transfer on a TCP connection may be delayed due to the influence of data transfer completion on other TCP connections. So, TCP connections should be managed individually in order to realize efficient delivery.

\section{3)Scheduling for lines}

The maximum number of TCP connections of multicast server $(M a x C)$ is pre-assigned considering the band width of the network, if the networks are the shared media type (ex. Ethernet LAN). If point - point network (ex. ISDN) are used, $\operatorname{Max} C$ should equal the number of supported lines by the multicast server. In the latter case, if the number of clients is greater than Max $C$, TCP connections can be established up to $\operatorname{Max} C$, simultaneously. Once file delivery completes on one TCP connection, MFTP tries to establish a new TCP connection for remaining clients.

\section{(2)Software configuration of Type A-1}

FTP is implemented as an application process in UNIX environments. In order to delivery the file to many clients, the number of FTP process in the multicast server should equal the number clients. This causes high CPU loads for dispatching the tasks in the multicast server. To minimize the number of processes, MFDF was realized as one application process. MFDF was implemented on SUN OS 4.1.3 and Solaris 2.3.

\subsection{Software configuration of Type B-1}

\subsubsection{Protocol Stack}

The protocol stack for Type B-1 is shown in Figure 2. IP multicast and UDP (Postel 1980) are selected as the network protocol and transport protocol, respectively. Both of these protocols are unreliable connectionless protocols. IP multicast realizes the multicast function using class D IP addresses in cooperate with the multicast router. UDP detects transmission error using checksum and if an error is detected in the packet, it is discarded.

RMTP and delivery application protocols are developed on top of UDP in order to supplement the reliable multicast delivery function. RMTP is a connection-mode transport protocol; it establishes and maintains the multicast connection during the data transfer phase, and realizes one-way reliable multicast data transmission. The delivery application protocol realizes user group management and information architecture control such as file architecture or document architecture.

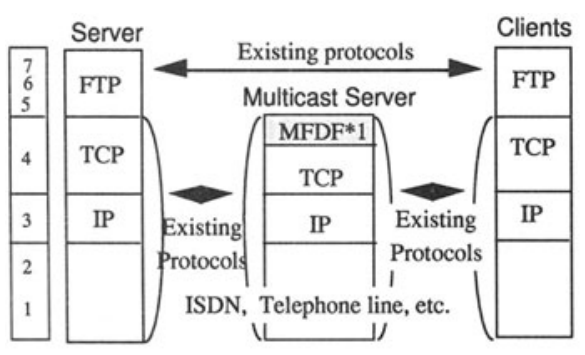

*1 MFDF:Multicast File Delivery Function

(a) Type A-1 Protocol Stack

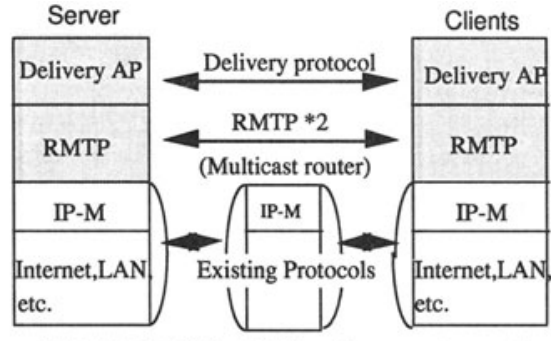

*2 RMTP: Reliable Multicast Transport Protocol

(b) Type B-1 Protocol Stack

Figure 2 Protocol Stacks of Type A-1 and Type B-1 


\subsubsection{Software for Type B-1}

\section{(1)Basic function of RMTP}

RMTP is a client - initiated protocol in which clients are responsible for detecting data packet loss and sending ACK/NACK to the server. The main function of RMTP is as follows: 1) Multicast connection management, 2)Error recovery control, 3)Flow control, 4)Packet scrambling, 5)Busy control, etc.

(2)Software configuration of Type B-1

RMTP and the delivery application are implemented on the socket interface supported by the UNIX platform as an application process. Both server and client software were implemented on Solaris 2.3.

\section{EVALUATION AND RESULTS}

\subsection{Evaluation environment}

To evaluate the Type A-1 tool, ISDN (CS, 64kb/s), and Ethernet LAN (10 M b/s) were used as the network. The server and multicast server were a work station (SUN Sparc Classic with $32 \mathrm{M}$ Bytes of memory : SUN OS 4.1.3, and SS 20 with $128 \mathrm{M}$ Bytes of memory : SUN OS 4.1.3). The clients were personal computers (PC-9821 AP2 with 16M Bytes of memory : Windows 3.1) for real clients, and work station (SUN Sparc2 with $32 \mathrm{M}$ Bytes of memory : SUN OS 4.1.3) for emulated clients. The file size was $1 \mathrm{M}$ Byte, packet size was $1 \mathrm{~K}$ Byte, and socket buffer size was $50 \mathrm{~K}$ Bytes.

The evaluation network for the Type B-1 tool consisted of three segmented Ethernet LANs with a multicast router (Baynetworks BCN router). The server was a work station (SUN SS 20 with $128 \mathrm{~K}$ Bytes of memory : Solaris 2.3), the clients were work stations (SUN SS 4, etc. with $32 \mathrm{M}-128 \mathrm{M}$ Bytes of memory : Solaris 2.3) for both real and emulated clients. The file size was $2 \mathrm{M}$ Bytes, packet size was $1 \mathrm{~K}$ Byte, and socket buffer size was 50 K Bytes.

\subsection{Results}

We evaluated the delivery time and server CPU load of both the conventional method (repeated $1: 1 \mathrm{FTP}$ ) and RIME.

\section{(1) Type A-1}

Server and multicast server CPU load and delivery time for Type A-1 are shown in Figure 3. In the case of 5,000 clients, Type A- 1 reduces CPU load by $90 \%$ compared to FTP, it also reduces total file transfer time by a factor of four to five times. These benefits were due to reductions in task switching and file $\mathrm{I} / \mathrm{O}$ overhead. One multicast server can deliver an $1 \mathrm{M}$ Byte file to 5,000 clients within 8 hours. This means that a delivery service can transfer an 1 M Byte file over midnight via public networks (ex. ISDN).

The main features of Type A-1 are as follows:

a)Suitable for medium-size file delivery to personal customers via public networks

b)Clients can use existing communication software (TCP/IP) without change

c)Cascade interconnection of multicast server significantly increases the number of clients supported

(2)Type B-1

d)Reduces server load and delivery time 
Server CPU load and delivery time for the Type B-1 tool are shown in Figure 4. In the case of 5,000 clients, Type B-1 reduces CPU load by a factor of $1 / 100$ compared to FTP. Delivery is 60 times faster. The performance of Type B-1 is acceptable for practical applications; for example, one server can deliver $2 \mathrm{M}$ Bytes (typical Japanese newspaper) to 5,000 clients within 3 minutes.

The main features of Type B-1 are as follows:

a)Suitable for medium - to large size file delivery to corporate users via Internet

b)Reduces network traffic using multicast routers

c)Reduces server load and delivery time drastically

d)Applicable to ATM point - multipoint connection

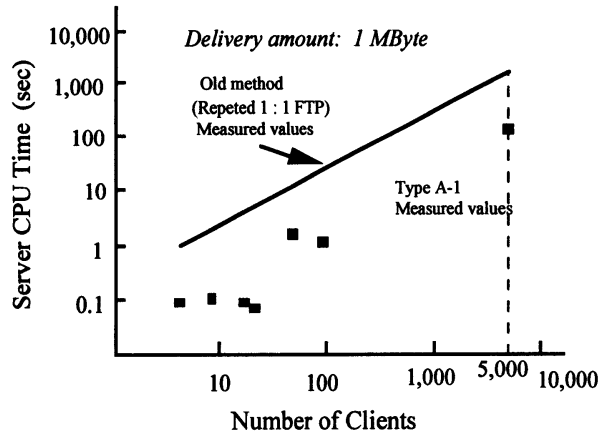

(a) Server CPU Load (Time)

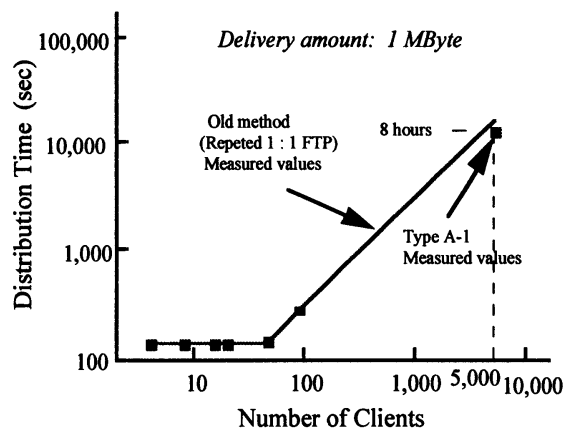

(b) Distribution Time

Figure 3 Performance of Type A-1

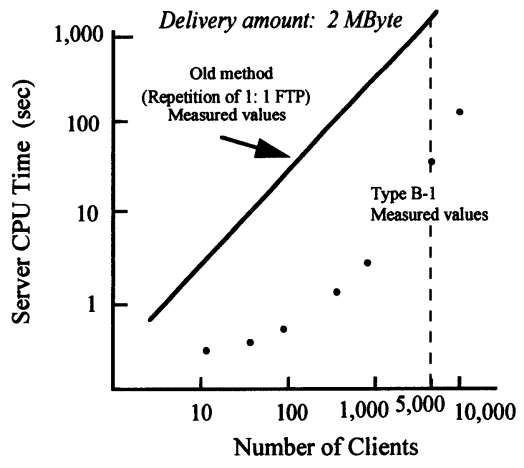

(a) Server CPU Load (Time)

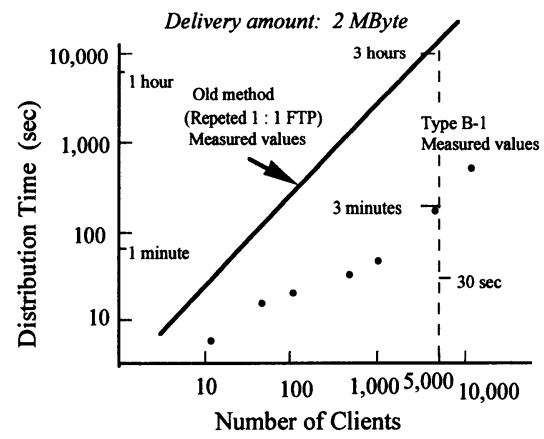

(b) Distribution Time

Figure 4 Performance of Type B-1

\section{CONCLUDING REMARKS}

We proposed RIME which realizes reliable information delivery service, and four tools. Two of them were evaluated by test networks, and the results confirmed the feasibility of RIME in realizing large scale information delivery.

RIME allows error-free and secure communications between a single server and thousands of remote clients. Using RIME it is possible to simultaneously multicast multimedia material 
such as data, text, image, video and even software updates over networks LAN, TCP/IP networks, ATM, ISDN, PSTN or the Internet. RIME confirms authorized clients before data transfer, and also confirms the (records) held by each client after data transfer.

RIME is going to be used in corporate information delivery system. Multicast operation issues such as dynamic group control, multicast routing, etc. are to be studied through field tests. RIME will work well for variety of services including distance learning and digital publication.

\section{REFERENCES}

Casner, S. and Deering, S. (1992) First IETF Internet Audiocast, ACM SIGCOMM CCR Vol.22, No.3

Postel, J. (1981) TRANSMISSION CONTROL PROTOCOL, RFC 793

Shiroshita, T., Takahashi, O. and Yamashita, M. et al. (1996) Reliable Multicast Transport

Protocol (RMTP) and its Applicability to Emerging Networks, TECHNICAL REPORT OF IEICE., SSE95-196, IN95-140

Deering, S.E. (1989) Host Extensions for IP Multicasting, RFC 1112

Postel, J. and Reynolds, J. (1985) FILE TRANSFER PROTOCOL (FTP), RFC 959

Postel, J. (1980) User Datagram Protocol, RFC 768 


\section{BIOGRAPHY}

Osamu Takahashi received M.S. degree in Information Engineering in Hokkaido University, in 1975. He is a senior research engineer at NTT Information and Communication Systems Labs., Yokosuka, Japan. He is engaging the development of multimedia communication protocols and their implementation. He is a member of the Information Processing Society of Japan.

Teruji Shiroshita received the B.S. and M.S. degrees in Applied Mathematics and Physics from Kyoto University, Kyoto, Japan, in 1982 and 1984. He is a Senior Research Engineer of the High-Speed Computer Networking Laboratory, NTT Information and Communication Systems Laboratories, Yokosuka, Japan. He has been engaged in research and development of multimedia, communication protocols and systems.

Tetsuo Sano received the B.E., M.E. degrees in information and computer sciences from Osaka University, Osaka, Japan, in 1992, 1994, respectively. In April 1994, he joined NTT, Japan. Currently he is working on High-Speed Computer Networking Laboratory, NTT Information and Communication Systems Laboratories, Yokosuka, Japan, as a researcher. He has been engaged in the implementation, evaluation, verification of communication protocols.

Masahide Yamashita received the B.S. and M.S. degrees from Tokyo University, Tokyo, Japan, in '72 and '74, respectively. He is the Executive Research Engineer of NTT Information and Communication Systems Labs. Since 1974 he has been with NTT Electrical Communications Labs, at Yokosuka, Japan. From 1974 to 1991 he was engaged in research and development of communication processors for DIPS computer systems which are widerange general purpose computer systems for NTT data communication services. Since 1991 he has been engaged in the research and development of high-speed computer networking technologies and equipments. He is a member of IEEE and ACM.

Yukihiro Nakamura received the B.S., M.S., and Ph.D. degrees in Applied Mathematics and Physics from Kyoto University, Kyoto, Japan, in 1967, 1969 and 1995, respectively. He is the Executive Manager of the High-Speed Computer Networking Laboratory, NTT Information and Communication Systems Laboratories, Yokosuka, and also a Guest Professor of Graduate School of Information Systems, University of Electro-Communications, Tokyo, Japan. Since 1969 he has been with the Electrical Communications Laboratories, NTT, at Tokyo, Yokosuka, and Kyoto, Japan. From 1969 to 1980 he was engaged in research and design of DIPS CPU hardware which was a general purpose large scale computer for NTT data communication services. Since 1981 he has been engaged in the research and development of high-level synthesis technology for parallel architecture design. He has developed a highlevel synthesis CAD system for ASIC design called "PARTHENON' which is one of the leading high-level CAD system in practical use in the world. Currently, he is an Associate Editor for the ACM TRANSACTIONS ON DESIGN AUTOMATION OF ELECTRONIC SYSTEMS (TODAES) and also IEEE TRANSACTIONS ON VLSI SYSTEMS. He was the Asian Representative of the ICCAD in 1991 and 1992. He has served as a member of the technical committee of the ICCAD, ED\&TC, ISSS and ASP-DAC. Dr. Nakamura received the Best Paper Award of IPSJ, the Okochi Memorial Technology Prize and the Minister Prize of the Science and Technology Agency in 1990, 1992, and 1994, respectively.

Masato Maruyama received the B.S.degree from Kanazawa University, Kanazawa, Japan. $\mathrm{He}$ is the General Manager of Multi-media System Group, NTT Electronics Technology Corporation, Kanagawa, Japan. Since 1986 to March,1996, he worked for the Electrical Communication Laboratories, NTT at Yokosuka, Japan. He was engaged in development of many kinds of communication control processors, and research of the communication systems for the multimedia services. He is engaging in development of Network Interface Card for ATM applications. 